\title{
Preoperative magnetic source imaging for brain tumor surgery: a quantitative comparison with intraoperative sensory and motor mapping
}

\author{
Hagen Schiffbauer, M.D., Mitchel S. Berger, M.D., Paul Ferrari, B.S., \\ Dirk Freudenstein, M.D., Howard A. Rowley, M.D., \\ And Timothy P. L. Roberts, Ph.D.
}

Department of Neurosurgery, University of Tübingen, Germany; and Departments of Radiology and Neurological Surgery, University of California at San Francisco, California

\begin{abstract}
Object. The aim of this study was to compare quantitatively the methods of preoperative magnetic source (MS) imaging and intraoperative electrophysiological cortical mapping (ECM) in the localization of sensorimotor cortex in patients with intraaxial brain tumors.

Methods. Preoperative magnetoencephalography (MEG) was performed while patients received painless tactile somatosensory stimulation of the lip, hand, and foot. The early somatosensory evoked field was modeled using a single equivalent current dipole approach to estimate the spatial source of the response. Three-dimensional magnetic resonance image volume data sets with fiducials were coregistered with the MEG recordings to form the MS image. These individualized functional brain maps were integrated into a neuronavigation system. Intraoperative mapping of somatosensory and/or motor cortex was performed and sites were compared.

In two subgroups of patients we compared intraoperative somatosensory and motor stimulation sites with MS imaging-based somatosensory localizations. Mediolateral projection of the MS imaging source localizations to the cortical surface reduced systematic intermodality discrepancies. The distance between two corresponding points determined using MS imaging and ECM was $12.5 \pm 1.3 \mathrm{~mm}$ for somatosensory-somatosensory and $19 \pm 1.3 \mathrm{~mm}$ for somatosensory-motor comparisons. The observed $6.5 \mathrm{~mm}$ increase in site separation was systematically demonstrated in the anteroposterior direction, as expected from actual anatomy. In fact, intraoperative sites at which stimulation evoked the same patient response exhibited a spatial variation of $10.7 \pm 0.7 \mathrm{~mm}$.

Conclusions. Preoperative MS imaging and intraoperative ECM show a favorable degree of quantitative correlation. Thus, MS imaging can be considered a valuable and accurate planning adjunct in the treatment of patients with intraaxial brain tumors.
\end{abstract}

\section{KEY WORDS • magnetoencephalography • brain mapping • neuronavigation • electrophysiological cortical mapping • magnetic source imaging • glioma • brain neoplasm}

Magnetic source imaging is becoming available at an increasing number of clinical centers worldwide as an adjunct in the planning and guidance of brain tumor surgery. ${ }^{1,15-19,24,27,33,39,44,46,47,55}$ It can be used to estimate the risk of incurring a neurological deficit while attempting surgically to remove intraaxial, supratentorial neoplasms or malformations. Thus, it is helpful in assessing surgical options, such as biopsy or partial or total resection, and their respective associated risks; in some cases, it may even lead to the decision for nonsurgical treatment. ${ }^{24,52}$ Furthermore, it enables the neurosurgeon to compare different surgical approaches more readily. ${ }^{1}$

Although randomized clinical trials have yet to be conducted, it appears that in treating most gliomas, an aggres-

\footnotetext{
Abbreviations used in this paper: $\mathrm{ECM}=$ electrophysiological cortical mapping; EMG = electromyography; $\mathrm{MEG}=$ magnetoencephalography; MR = magnetic resonance; MS = magnetic source; SED = single-equivalent current dipole; $2 \mathrm{D}=$ two-dimensional; $3 \mathrm{D}=$ three-dimensional.
}

sive resection improves the patient's chance of survival and postoperative quality of life if neurological function can be preserved. ${ }^{2-5,13,28,60}$ Preoperatively acquired individual functional maps of the brain, derived, for example, using MS imaging, can be integrated into neuronavigation systems to guide interactively the surgeon during tumor resection, thus assisting him or her in the identification and preservation of eloquent cortex..$^{1,15,19,44,47}$

If the decision to perform a craniotomy has been made, however, the use of intraoperative ECM is still deemed mandatory if the tumor is encroaching in the vicinity of functional brain area. ${ }^{5,7,8,34,40,63}$ Intraoperative ECM is considered to be the gold standard in the neurosurgical determination of sensory, motor, and language-related cortex, with which any noninvasive method must be compared both qualitatively and, important in this study, quantitatively.

Especially for intraoperative language cortex mapping but also for somatosensory cortex mapping, awake craniotomy techniques have regained popularity during the last 
TABLE 1

Distribution of histological diagnoses*

\begin{tabular}{lc}
\hline \hline \multicolumn{1}{c}{ Histological Diagnosis } & No. of Patients \\
\hline ganglioglioma & 7 \\
astrocytoma (Grade 2 or 3) $\dagger$ & 45 \\
oligoastrocytoma (Grade 2 or 3) $\dagger$ & 52 \\
oligodendroglioma (Grade 2 or 3) $\dagger$ & 31 \\
glioblastoma multiforme & 55 \\
PNET & 3 \\
metastasis & 5 \\
astrogliosis & 6 \\
miscellaneous lesion $\ddagger$ & 17 \\
total§ & 221 \\
\hline
\end{tabular}

$*$ PNET $=$ primitive neuroectodermal tumor.

$\dagger$ Tumor grades were determined based on the St. Anne-Mayo classification system developed by Daumas-Duport.

\$ This group includes those diagnoses that were encountered only once or twice.

$\S$ Three patients in this series of 224 did not undergo surgery.

decade as new intravenous sedative and narcotic agents have made the procedure safer and more comfortable for the patient, allowing for earlier discharge of patients from hospitals and thus decreased hospitalization costs. ${ }^{10,34,36,40,57}$

Nevertheless, there is substantial evidence for the usefulness of MS imaging and its qualitative reliability in the localization of functional areas such as the rolandic cortex. Even details of the human homunculus, as first described by Penfield $^{41}$ in 1937, can be mapped using MEG in individual volunteers and patients. $31,38,52,62$ The quantitative localization accuracy of MS imaging has not been adequately verified in a larger group of patients, however. In the present study we addressed this issue through spatial comparison of preoperative MS imaging source localizations and intraoperative ECM sites.

\section{Clinical Material and Methods}

\section{Patient Population}

Between December 1995 and November 2001, a total of 224 patients (128 male and 96 female) harboring supratentorial, intraaxial brain lesions were referred in-house to the Biomagnetic Imaging Laboratory to undergo preoperative MS imaging. Ages of the patients ranged from 13 to 82 years, with a mean age of 42 years. Tumors were located in the left hemisphere in 151 patients, in the right hemisphere in 72, and in both hemispheres in one patient. Some of the patients had been referred to the Department of Neurological Surgery at the University of California at San Francisco by other neurosurgeons because their tumors were located in the vicinity of functionally important cortex and an intraoperative ECM procedure had been deemed necessary to avoid incurring a surgically induced neurological deficit. The patients' diagnoses are listed in Table 1, showing a wide variety of typical intraaxial neoplastic and nonneoplastic lesions. The miscellaneous lesions group consists of those diagnoses that were encountered only once or twice. Three patients did not undergo surgery as a result of preoperative MS imaging, and thus a histological diagnosis could not be provided. All research procedures were performed in accordance with the University of California at San Francisco Committee on Human Research.

\section{Magnetic Source Imaging}

Noninvasive mapping of the somatosensory cortex was achieved using a dual 37-channel biomagnetometer. Magnetoencephalography was usually performed 2 days prior to surgery on an outpatient basis to allow ample time for discussion of the results and implications. The biomagnetometer was housed in a magnetically shielded room constructed from layers of aluminum and $\mu$-metal, an alloy of extremely high magnetic permeability, designed to occlude extraneous electric and magnetic fields. The room is approximately $4 \times 4 \times 3 \mathrm{~m}$ in dimension, and although it is enclosed, none of our patients experienced claustrophobia. Painless somatosensory stimulation was administered as in previous studies $47,52,62$ by using a compressed air-driven diaphragm clip (15-20 psi) attached to the fingertips, lips, and toes. Stimulation was consequent to the application of pulses of 30-msec duration; 256 such stimulations were applied at pseudorandom interstimulus intervals of 400 to $600 \mathrm{msec}$. Epochs of 300-msec duration were collected with a high-pass filter (cutoff $1 \mathrm{~Hz}$ ) for each stimulus event at a sampling rate of 298 to $1041 \mathrm{~Hz}$, beginning at 150 msec prestimulus to establish baseline activity. Stimuluslocked averaging was used to improve signal-to-noise ratio. The averaged MEG epoch for each detector channel was then digitally filtered with an 8 - to $40-\mathrm{Hz}$ passband to exclude high-frequency noise before source modeling.

The SED model was used to identify the presumed neuronal source. Single equivalent dipole modeling based on the spherical head assumption ${ }^{22,51}$ is simplest computationally and proves effective for simple sensory stimuli that elicit focal activity in groups of cortical neurons organized tangentially to the scalp surface, and for early poststimulus neuronal activity. The SED that provided the strongest detected signal and that best satisfied the single dipole model within the poststimulus latency range from 30 to $70 \mathrm{msec}$ was chosen to represent the neuronal source in the final data set. To accept a source localization, solutions were required to show root-mean-square-detected magnetic fields on the order of 50 femtoteslas, data-model correlations with an $\mathrm{r}$ greater than 0.98 , and a minimal $95 \%$ statistical confidence volume.

Registration of the MEG-derived sources with high-resolution anatomical images to form the final MS images was achieved using a triangulation system with fixed receivers and a stylus transmitter placed on external landmarks, such as the nasion and preauricular points, and subsequently identifying these landmarks on 3D MR images. Depending on the number of stimulation sites and paradigms, the entire MEG examination could be performed within 1 to 2 hours, with reconstruction, dipole fitting, MR image overlaying, and filming requiring an additional 1 to 2 hours.

High-resolution MR images were acquired using a 1.5tesla MR imager to provide the necessary anatomical detail for surgical planning and neuronavigation. The protocol typically included the following sequences: 1) a $\mathrm{T}_{1}$-weighted, 3D spoiled gradient-recalled echo in a steady state with sequence TR $34 \mathrm{msec}$, TE 3 to $8 \mathrm{msec}$, flip angle $30^{\circ}$, slice thickness $1.5 \mathrm{~mm}$, matrix $256 \times 256 \times 124$, and field of view $260 \times 260 \mathrm{~mm}$ with skin-to-skin coverage to include the nasion, preauricular points, and external fiducial markers; 2) a $\mathrm{T}_{2}$-weighted 3D fast-spin echo sequence with TR $3000 \mathrm{msec}$, TE $105 \mathrm{msec}$, slice thickness $1.5 \mathrm{~mm}$, and ma- 
trix $256 \times 256 \times($ range $108-132)$; and 3 ) sagittal $\mathrm{T}_{1}$ weighted and a coronal fluid-attenuated inversion recovery sequences, included for diagnostic purposes. Magnetic resonance images were transferred through the hospital computer network to the MEG workstations, and from there, after fusion with the MEG dipoles, to the workstation of the neuronavigation system in the operating room.

\section{Surgical Procedure, Neuronavigation, and Intraoperative Monitoring}

Patients underwent craniotomies while awake in 120 cases $(54.3 \%)$ and under general anesthesia in 101 cases (45.7\%). During the series we performed seven open biopsies and one burr hole biopsy. Awake craniotomies were reserved mainly for those patients who underwent mapping of the language-related cortex in addition to mapping of the motor cortex at its junction with the sylvian fissure. Note that three patients did not undergo surgery.

Neuronavigation was performed using the Stealth Station in 175 patients $(79.2 \%)$, the Viewing Wand in 33 (14.9\%), and two other commercially available systems in five $(2.3 \%)$; neuronavigation was unavailable or deemed unnecessary in eight cases (3.6\%). The navigation system was used to plan a well-centered craniotomy and to expose functional cortical areas as much as needed to achieve a radical but function-preserving resection. The registration accuracy of the Stealth Station was typically approximately $0.8 \mathrm{~mm}$ with the use of 10 fiducial points and a subsequent surface merge. As an upper limit of the registration accuracy, we accepted $1.2 \mathrm{~mm}$.

For awake procedures patients were positioned comfortably, with all extremities well padded such that the intended craniotomy and tumor resection could be appropriately performed and the anesthesiologist had an unobstructed view of and easy access to the patient. Patients were secured with pin fixation. A heating blanket was kept on the patient to maintain his or her core temperature to within $1^{\circ} \mathrm{C}$ of normal. If the patient's temperature drifted too low, stimulation mapping of the cortex became difficult. A local anesthetic agent consisting of a mixture of lidocaine $(0.5 \%)$, bupivacaine $(0.25 \%)$, and epinephrin in bicarbonate solution was applied to the patient's scalp. A continuous propofol infusion was used to maintain a hypnotic state on completion of dural exposure. The patient's head was positioned in a Mayfield headrest and the neuronavigation system was set up. Details on the awake procedures may be found in our previous publications. ${ }^{6,34}$ For patients who did not require language mapping, general anesthesia was maintained using a 70:30\% mixture of nitrous oxide/oxygen supplemented with isoflurane $(0.25-0.5 \%$ end-tidal concentration) and fentanyl (1-4 $\mu \mathrm{g} / \mathrm{kg})$. Propofol (0-100 $\mu \mathrm{g} / \mathrm{kg} / \mathrm{min})$ was administered as a supplement if necessary.

After craniotomy and dural opening, in a majority of cases the cortex was mapped to define the borders of the motor region before tumor resection. We used the Ojemann cortical stimulator with a handheld bipolar electrode that had 5-mm spacing between the tips. It produced a train of square-wave, biphasic pulses of 1-msec duration at a frequency of $60 \mathrm{~Hz}$. The stimulus current required to produce a motor response usually ranged from 2 to $16 \mathrm{~mA}$. The current was increased in 1- to $2-\mathrm{mA}$ increments until a response was obtained (up to a maximum level of $8 \mathrm{~mA}$ in the awake patient and $16 \mathrm{~mA}$ in the anesthetized patient). Stimulation was applied for approximately 1 second each time. If the expected location of the sensorimotor cortex was at the border of the craniotomy, a strip electrode was inserted subdurally to map the hidden cortex. A multichannel EMG recording was obtained using 12 to 16 subdermal, intramuscular needle electrodes on a Nicolet Viking IV P system or, if unavailable, by using a set up consisting of Grass P511J amplifiers (bandpass 30-1000 Hz) and a six-channel storage oscilloscope. ${ }^{63}$ Additionally, the anesthesiologist observed the patient carefully. If the patient was awake, he or she was asked to provide information on sensations, and language capabilities were tested according to a previous-

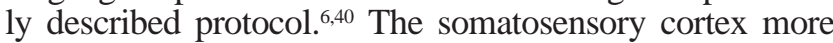
readily produced a stimulus response, requiring a less intense electrical current to evoke a response than that used during motor mapping. If movement or EMG activity persisted after removal of the stimulating electrode, cold, lactated Ringer solution was applied to the exposed cortex; if this was insufficient, methohexital was administered to terminate the activity. ${ }^{50}$

Depending on the location of the tumor, subcortical stimulation was sometimes used to identify the corona radiata and the internal capsule at the border of the resection area. Before closure of the dura mater, previously marked cortical sites were usually restimulated to confirm the integrity of the motor pathways. Sites at which a positive response could be elicited were marked with a ticket. In a subgroup of patients, the precise cortical surface anatomical location of the site was also recorded using the neuronavigation system by placing the pointer at the site and performing a screen-save operation of the presented axial and coronal MR images, with the location of the pointer itself indicated by superimposed crosshairs.

\section{Magnetic Source Imaging Analysis}

Using a neuronavigation system, a total of 201 screen snapshots were acquired intraoperatively in 54 patients and saved for documentation purposes and post hoc analysis. In 43 of these patients, corresponding preoperative MS imaging source localizations and intraoperative mapping sites could be identified. The localization of the crosshair on the neuronavigation system snapshot was visually identified and marked on the corresponding axial MS image, and its $\mathrm{x}-, \mathrm{y}-$, and $\mathrm{z}$-coordinates were determined using MRVision software. The coordinates of the center of the corresponding MEG source could be readily identified on the MS images. Reproducibility of this process was within one pixel in both the anteroposterior and mediolateral directions. Because in some cases the neuronavigation system was used to calculate intermediate axial slices, localization precision in the superoinferior direction was half of one slice thickness $(0.75 \mathrm{~mm})$. In a second step, a mediolateral projection of the MS imaging source localization to the cortical surface was performed, reducing systematic intermodality discrepancies and thus leading to a 2D point-to-point distance.

A limited number of anatomical sites were stimulated during preoperative MS imaging. Typically, they included digits, toes, and lips. Intraoperative motor responses such as movement of the lip, mouth, jaw, face, tongue, throat, or pharynx were accepted for comparison with somatosensory MS imaging source localization of the corresponding side 


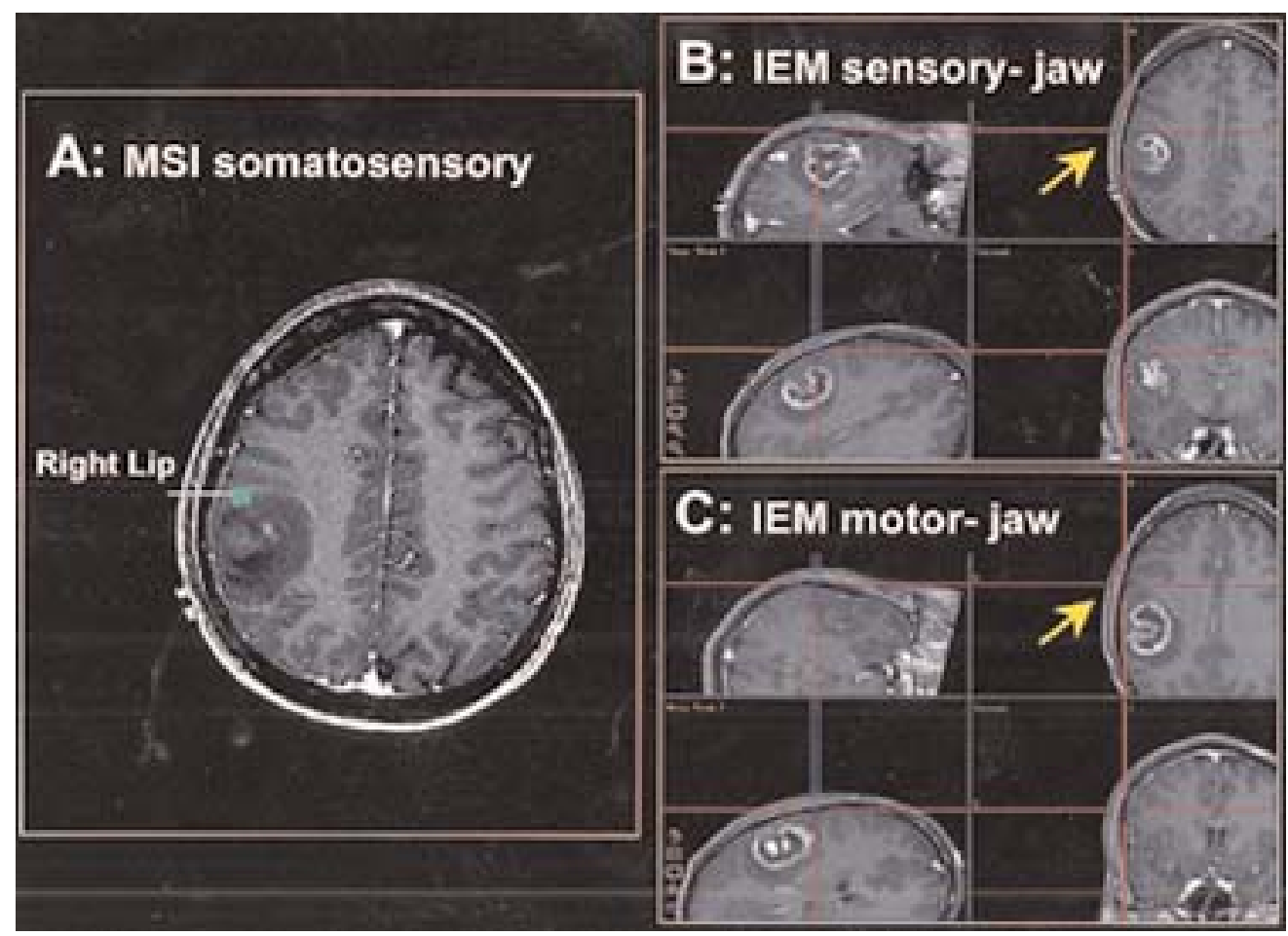

FIG. 1. Case 1. This 44-year-old left-handed man had experienced intermittent numbness and tingling in his right upper extremity, face, and right chest during a period of 2 months. He also reported some word-finding difficulties. Preoperative neurological examination was entirely normal. On MR imaging a $4.3 \times 3.2-\mathrm{cm}$, rim-enhancing lesion was demonstrated in the left temporoparietal region. During an awake craniotomy procedure supported by intraoperative ECM (IEM), a gross-total resection of the mass was achieved. No new neurological deficit was incurred, and the patient was discharged from the hospital in a stable condition on the third postoperative day. A: A $\mathrm{T}_{1}$-weighted, contrast-enhanced MR image revealing a modeled neuronal source on the margin of the hypointense area of this glioblastoma multiforme located in the left temporoparietal area. The SED modeled source (green square) labeled "Right Lip" corresponds to the site of tactile stimulation of the right side of the lip. The source localization is more superior than expected from anatomy, which is indicative of a displacement due to the pathological mass. Magnetic source imaging localization and intraoperative ECM sites were separated by $12.4 \mathrm{~mm}(3 \mathrm{D})$ and $5.4 \mathrm{~mm}(2 \mathrm{D})$ after mediolateral projection. B: Intraoperatively obtained neuronavigation system screen snapshots of one ECM site that elicited sensation in the jaw. The 3D distance between this site and other ECM sites (not shown) was 9.1 and $14.1 \mathrm{~mm}$. Two factors potentially contribute to these findings: 1) the extent of cortical representation of the lip in the postcentral gyrus; and 2) the spread of electrical current from the bipolar stimulation. C: Intraoperatively obtained neuronavigation system screen snapshots of an ECM site that elicited movement of the jaw. This and another ECM site (not shown) were separated by $12.1 \mathrm{~mm}$. Both sites were located one gyrus anterior to the sites eliciting sensation in the jaw (B).

of the lip. Stimulation-induced movements of the forearm, hand, or singular digits were compared with somatosensory MS imaging source localizations of one of the digits. The closest match for each anatomical area was included in the final analysis, accounting for 38 comparisons in 37 patients.

Similarly, somatosensory sensations of the lip, mouth, tongue, jaw, or throat were accepted for comparison to somatosensory MS imaging source localization of the corresponding side of the lip. Sensations in a single digit or several fingers were compared with somatosensory MS imaging source localizations of one of the digits. Again, the closest match for each anatomical area was included in the final analysis, accounting for 34 comparisons in 28 patients.

A two-tailed t-test was applied to calculate the significance of the observed shift in the anteroposterior direction between somatosensory (MS imaging)-somatosensory (ECM) and somatosensory (MS imaging)-motor (ECM).

In 18 patients, 30 comparisons were possible between two intraoperatively derived mapping points that had elicit- ed exactly the same somatosensory (13 comparisons) or motor (17 comparisons) response. Similar to the intermodality comparison above, the 2D and 3D intramodality displacements were determined to define the extent of the cortex from which a certain movement or sensation could be provoked with the applied ECM method and, in effect, the practical variation in the spatial site location determined using intraoperative ECM.

\section{Sources of Supplies and Equipment}

The biomagnetometer (Magnes II) including its software for analysis was acquired from 4D Neuroimaging (San Diego, CA), the Stealth Station Treatment Guidance System from Medtronic Sofamor Danek (Broomfield, CO), and the Viewing Wand from ISG Technologies (Toronto, ON, Canada). The 1.5-tesla MR imager (Signa) was manufactured and regularly upgraded by General Electric Medical Systems (Milwaukee, WI). The Nicolet Viking IV P system 
was obtained from Nicolet Biomedical (Madison, WI), the amplifiers (Grass P511J) from Grass Instruments/AstroMed (West Warwick, RI), and the Ojemann cortical stimulator (model OCS-1) from Radionics (Burlington, MA). The MRVision software was acquired from The MRVision Co. (Winchester, MA).

\section{Results}

\section{Patient Outcome}

For all 221 patients who underwent surgery, the digital hospital records were screened for information on preoperative symptoms and signs and intraoperative mapping success as well as new neurological deficits in the early postoperative period and at discharge. Changes in neurological condition were analyzed separately for sensorimotor function and other neurological deficits such as impairment of language. The condition of the patient was classified as improved, unchanged, having a new transient deficit that was reversed at the time of discharge, having a new deficit that showed considerable improvement during the postoperative period prior to discharge, having new mild deficits, or having new considerable deficits without signs of improvement.

\section{Technical Success of MS Imaging}

Noninvasive somatosensory mapping using MEG was more than $97 \%$ technically successful with the digits of the hand for both tumor-affected and unaffected hemispheres. Lip localization was possible in more than $90 \%$ of cases. More challenging was the localization of somatosensory cortical representation of the toes. Localization was not attempted in $56 \%$ of cases, but it was successful in $82 \%$ of attempted cases. In all but two patients at least one MS imaging source localization was noted in the hemisphere affected by the tumor. Patients who had undergone previous surgery or exhibited excessive dental amalgam or other metal hardware in their body were prone to more serious artifacts, necessitating exclusion of obviously aberrant MEG channels.

\section{Results of Quantitative Comparison}

The quantitative comparison of somatosensory preoperative MS imaging source localizations and intraoperative ECM sites (Fig. 1) led to the following results. The 3D distance between somatosensory sites on MS imaging and somatosensory sites on intraoperative ECM was a mean of $20.5 \pm 1.5 \mathrm{~mm}$ (Table 2). The minimum distance observed was $1.5 \mathrm{~mm}$ and the maximum distance, $42.2 \mathrm{~mm}$. To eliminate a systematic intermodality discrepancy, a mediolateral projection of the MS imaging source localization was calculated. The consequent 2D distance for the same pair of sites was a mean $12.5 \pm 1.3 \mathrm{~mm}$ (range $1.5-30.3 \mathrm{~mm}$ ). The 3D distance between somatosensory sites on MS imaging and motor sites on intraoperative ECM was $22.8 \pm 1.6 \mathrm{~mm}$ (range 5.3-49.1 $\mathrm{mm}$ ); the corresponding 2D distance was $19 \pm 1.3 \mathrm{~mm}(4.3-38.4 \mathrm{~mm})$. The mean difference in the anteroposterior direction was $6.7 \pm 1.4 \mathrm{~mm}$ for somatosensory sites on ECM compared with somatosensory sites on MS imaging, and $12.9 \pm 1.7 \mathrm{~mm}$ for motor sites on ECM compared with somatosensory sites on MS imaging. Note
TABLE 2

Quantitative comparison of intraoperative somatosensory mapping sites and preoperative somatosensory MS imaging sites*

\begin{tabular}{|c|c|c|c|c|}
\hline \multirow[b]{2}{*}{ Case No. } & \multirow{2}{*}{$\begin{array}{l}\text { Intraop } \\
\text { Sensation }\end{array}$} & \multirow{2}{*}{$\begin{array}{l}\text { MS Imag- } \\
\text { ing Stimula- } \\
\text { tion Site }\end{array}$} & \multicolumn{2}{|c|}{ Distance $(\mathrm{mm})$} \\
\hline & & & $3 \mathrm{D}$ & $2 \mathrm{D}$ \\
\hline 1 & tongue & lip & 10.1 & 10.1 \\
\hline 3 & $\operatorname{Dg} 1$ & $\operatorname{Dg} 2$ & 20.5 & 3.0 \\
\hline 3 & jaw & lip & 10.1 & 4.3 \\
\hline 4 & mouth & lip & 42.2 & 21.8 \\
\hline 6 & lip & lip & 31.6 & 17.3 \\
\hline 7 & lip & lip & 15.1 & 4.9 \\
\hline 7 & Dg1 & Dg1 & 25.8 & 10.9 \\
\hline 9 & jaw & lip & 12.4 & 5.4 \\
\hline 10 & tongue & lip & 22.8 & 21.3 \\
\hline 11 & mouth & lip & 19.5 & 10.9 \\
\hline 13 & lip & lip & 20.5 & 9.3 \\
\hline 14 & tongue & lip & 24.4 & 24.4 \\
\hline 16 & tongue & lip & 29.3 & 21.1 \\
\hline 17 & $\mathrm{Dg} 4 / 5$ & $\operatorname{Dg} 5$ & 12.8 & 11.3 \\
\hline 17 & $\operatorname{Dg} 1$ & $\operatorname{Dg} 2$ & 18.1 & 17.8 \\
\hline 20 & mouth & lip & 12.6 & 9.6 \\
\hline 21 & throat & lip & 28.7 & 16.6 \\
\hline 22 & mouth & lip & 32.7 & 30.3 \\
\hline 23 & finger-palm & Dg5 & 20.1 & 18.3 \\
\hline 24 & tongue & lip & 19.7 & 7.2 \\
\hline 26 & mouth & lip & 33.0 & 26.1 \\
\hline 28 & lower lip & lip & 29.3 & 14.5 \\
\hline 29 & finger & $\operatorname{Dg} 5$ & 1.5 & 1.5 \\
\hline 29 & $\operatorname{Dg} 1$ & $\operatorname{Dg} 1$ & 14.4 & 2.5 \\
\hline 30 & Dg1 & Dg1 & 26.2 & 6.3 \\
\hline 33 & Dg1 & $\operatorname{Dg} 1$ & 19.4 & 1.8 \\
\hline 33 & lip & lip & 11.9 & 4.1 \\
\hline 34 & Dg1 & $\operatorname{Dg} 1$ & 20.7 & 9.7 \\
\hline 34 & mouth & lip & 10.2 & 6.1 \\
\hline 38 & tongue & lip & 14.6 & 13.3 \\
\hline 40 & tongue & lip & 21.4 & 19.4 \\
\hline 41 & tongue & lip & 31.3 & 18.3 \\
\hline 42 & lower lip & lip & 17.1 & 13.0 \\
\hline 43 & tongue & lip & 15.6 & 12.7 \\
\hline mean $\pm \mathrm{SE}$ & & & $20.5 \pm 1.5$ & $12.5 \pm 1.3$ \\
\hline
\end{tabular}

$* \mathrm{Dg}=$ digit $\mathrm{SE}=$ standard error.

that MS imaging source localizations were posterior to the ECM sites (Table 3). These displacements were systematically different $(\mathrm{p}<0.01)$. The observed $6.5 \mathrm{~mm}$ mean difference in the 2D distance between the two measurement methods can be attributed to the fact that the somatosensory cortex in the postcentral gyrus lies posterior to the motor cortex in the precentral gyrus.

Sites that, when stimulated intraoperatively, elicited exactly the same motor or somatosensory response were located $11.3 \pm 0.7 \mathrm{~mm}(3 \mathrm{D})$ and $10.7 \pm 0.7 \mathrm{~mm}(2 \mathrm{D})$ apart. As expected, 2D and 3D distances were not significantly different from each other $(p>0.05)$. And, as might be expected from the principal direction of the rolandic cortex, the major portion $(8.7 \mathrm{~mm})$ was in the superoinferior direction.

\section{Electrophysiological Cortical Mapping and Patient Outcome}

Intraoperative ECM of the motor cortex was attempted in 170 cases (76.9\%), and of these it was successful in 147 $(86.5 \%)$. Data on early postoperative neurological status and status at discharge from the hospital were available 
H. Schiffbauer, et al.

TABLE 3

Quantitative comparison of intraoperative motor mapping sites and preoperative somatosensory MS imaging sites

\begin{tabular}{|c|c|c|c|c|}
\hline \multirow[b]{2}{*}{ Case No. } & \multirow{2}{*}{$\begin{array}{l}\text { Intraop } \\
\text { Sensation }\end{array}$} & \multirow{2}{*}{$\begin{array}{l}\text { MS Imag- } \\
\text { ing Stimu- } \\
\text { lation Site }\end{array}$} & \multicolumn{2}{|c|}{ Distance (mm) } \\
\hline & & & $3 \mathrm{D}$ & $2 \mathrm{D}$ \\
\hline 1 & mouth & lip & 7.4 & 6.8 \\
\hline 2 & face & lip & 15.4 & 11.6 \\
\hline 3 & jaw & lip & 16.3 & 16.3 \\
\hline 4 & tongue & lip & 49.1 & 38.4 \\
\hline 5 & face & lip & 40.8 & 22.7 \\
\hline 6 & mouth & lip & 37.6 & 29.4 \\
\hline 7 & finger flexion & Dg1 & 22.6 & 19.6 \\
\hline 8 & pharynx & lip & 32.6 & 25.4 \\
\hline 9 & jaw & lip & 15.2 & 14.3 \\
\hline 10 & mouth & lip & 35.5 & 33.7 \\
\hline 11 & pharynx & lip & 28.2 & 25.4 \\
\hline 12 & mouth & lip & 12.0 & 11.8 \\
\hline 13 & hand & Dg1 & 20.5 & 16.5 \\
\hline 14 & throat & lip & 22.5 & 21.7 \\
\hline 15 & face & lip & 24.9 & 17.9 \\
\hline 17 & mouth & lip & 19.4 & 15.9 \\
\hline 18 & jaw & lip & 18.7 & 9.3 \\
\hline 19 & hand-forearm & Dg1 & 23.0 & 22.9 \\
\hline 20 & mouth & lip & 21.1 & 20.2 \\
\hline 21 & throat & lip & 33.9 & 26.4 \\
\hline 23 & Dg1 & Dg1 & 8.3 & 7.7 \\
\hline 24 & hand-forearm & Dg5 & 9.2 & 9.2 \\
\hline 25 & throat & lip & 26.2 & 18.7 \\
\hline 26 & mouth & lip & 32.7 & 29.4 \\
\hline 27 & $\operatorname{Dg} 1 / \operatorname{Dg} 2$ & $\operatorname{Dg} 2$ & 20.5 & 18.4 \\
\hline 29 & jaw & lip & 25.2 & 24.4 \\
\hline 31 & face-eyebrow & lip & 5.3 & 4.3 \\
\hline 31 & finger extension & $\operatorname{Dg} 2$ & 16.8 & 12.6 \\
\hline 32 & hand & $\operatorname{Dg} 5$ & 15.1 & 13.3 \\
\hline 34 & mouth & $\operatorname{lip}$ & 17.1 & 15.5 \\
\hline 35 & mouth & $\operatorname{lip}$ & 29.0 & 22.5 \\
\hline 36 & hand & $\operatorname{Dg} 5$ & 22.2 & 16.1 \\
\hline 37 & mouth & lip & 23.8 & 22.0 \\
\hline 38 & mouth & lip & 9.7 & 9.2 \\
\hline 39 & lip & lip & 35.0 & 31.5 \\
\hline 40 & mouth & lip & 24.9 & 23.1 \\
\hline 41 & mouth & lip & 27.5 & 19.6 \\
\hline 42 & jaw & lip & 22.7 & 18.5 \\
\hline mean $\pm \mathrm{SE}$ & & & $22.8 \pm 1.6$ & $19.0 \pm 1.3$ \\
\hline
\end{tabular}

in 201 of 221 patients who underwent surgery. Changes in neurological status with respect to motor and somatosensory function comparing the pre- and postoperative situations are listed in Table 4. Overall changes in neurological status, taking into account speech and language deficits and visual field defects, are shown in Table 5. Eighty-one percent of the patients in this series left the hospital in an unchanged or even an improved condition despite the radical tumor resection strategy. Patients were usually discharged 3 to 5 days after surgery.

Within this early postoperative period, 23 patients (11.4\%) showed considerable improvement in their new deficit. Many of them were expected to return to full neurological function within the rehabilitation phase to follow. In only six patients (3\%), a new considerable deficit was incurred that showed no sign of improvement in the early postoperative period (that is, by the time of discharge from the hospital). The extent of resection was documented on early postoperative MR images acquired usually within the first 48 hours after surgery. Comparing operative and neu-
TABLE 4

Patient outcome with regard to motor and somatosensory functions

\begin{tabular}{lc}
\hline \hline \multicolumn{1}{c}{ Change in Neurological Status } & No. of Patients (\%) \\
\hline improved & $10(5)$ \\
no new deficit/unchanged & $165(82)$ \\
new transient deficit & $6(3)$ \\
new deficit but improvement prior to discharge & $16(8)$ \\
new considerable deficit w/ no change prior to discharge & $4(2)$ \\
total & $201(100)^{*}$ \\
\hline
\end{tabular}

* Data on changes in neurological status were not available in 20 patients, and three patients in this series did not undergo surgery.

roradiological reports, a gross-total resection of the enhancing tumor mass for enhancing lesions and of the hyperintense area on $\mathrm{T}_{2}$-weighted images for nonenhancing lesions was almost always achieved when intended. Typically, any residual tumor was found on images in the area that had been left untouched during surgery because intraoperative ECM demonstrated involvement of functional cortex or subcortical pathways.

Of the 224 patients who were referred for preoperative MS imaging, three (1.3\%) did not undergo surgery and eight $(3.6 \%)$ were selected for a biopsy procedure.

\section{Discussion}

The main finding of this study was that MS imaging provides accurate localization of the somatosensory cortex during neurosurgical planning in patients with critically located brain tumors, with a mean distance to ECM stimulation sites of $12.5 \mathrm{~mm}$. Corresponding motor cortex sites were found at a mean distance of $19 \mathrm{~mm}$, with the additional $6.5 \mathrm{~mm}$ difference in distance attributable mainly to the more anterior localization of the precentral (motor) gyrus compared with the postcentral (somatosensory) gyrus. Given the observation that multiple stimulation sites (mean separation $10.7 \mathrm{~mm}$ ) led to the same intraoperative responses, the discrepancy between MS imaging and ECM appears comparable to the magnitude of the uncertainty of each individual method.

\section{Technical Aspects of MS Imaging}

In a clinical setting, it is important to achieve a reasonable compromise between mapping all parts of the somatosensory homunculus and applying a protocol that is of sufficiently short duration and subjectively tolerable to achieve good patient compliance. Stimulating the lips, fingers, and toes and recording over both hemispheres spans most of the homunculus, includes the most robust sources of neuronal activity, and limits the undertaking to a period of 1 to 2 hours of somatosensory MEG mapping. We confirmed the finding of Nakamura, et al., ${ }^{38}$ that from this chosen set of stimuli, source localizations of the fingers were readily identified in more than $97 \%$ of cases and those of toes were least reliably mapped (82\%). Nakamura, et al., mapped the complete somatosensory homunculus in healthy volunteers and described even more difficulties in localizing the cortical representation of other body parts, for example, the forearm, knee, or ankle.

In this report we restricted comparisons to somatosen- 
sory MS imaging. Motor organization can be studied using MEG by recording voluntary finger motion; however, MEG responses to motor task performance present an extraordinary challenge to SED modeling. A fraction of patients from the present study population underwent an additional hand motor task (unpublished data). In accordance with earlier findings, ${ }^{58}$ the motor data were generally not as reproducible or precisely located as the somatosensory mapping data. Additionally, in patients presenting with a motor deficit, an MEG of motor responses might not technically be feasible in as many as $21 \%$ of cases. ${ }^{29}$ In conclusion, for presurgical evaluation of our clinical population, we adopted the more robust method of somatosensory MS mapping and made inferences about the location of motor cortex based on knowledge of somatosensory localization.

\section{Accuracy and Precision of MS Imaging}

The accuracy of MS imaging is dependent on a number of factors, including the sensitivity to certain components of the magnetic field, the cortical area activated by a stimulus applied to the somatosensory region, and the registration accuracy when overlaying MEG source localizations on MR images. Finally, the use of MS imaging data from a neuronavigation system confers additional sources of error during clinical application.

Practically, this recording property optimizes MEGbased detection of sources that are located in the bank of a gyrus in the depth of a sulcus, while minimizing sensitivity to sources in the tips of that gyrus. Intraoperative ECM, conversely, elicits sites at the brain surface. Thus, a direct 3D correlation of MS imaging source localizations and ECM sites leads to a systematic intermodality discrepancy mediolaterally. Therefore, in previous studies a variety of stratifying projection techniques have been used..$^{20,33,47}$

It has been estimated that at least 30,000 neurons must be simultaneously activated for current biomagnetometers to detect resulting fields..$^{59}$ Nakamura, et al. ${ }^{38}$ calculated that the primary somatosensory cortex area activated by stimulating the thumb and lower lip would be 93 and $68 \mathrm{~mm}^{2}$, respectively. These values were based on a hypothesis put forward by Chapman, et al., ${ }^{9}$ that a dipole moment of 10 nAm corresponded to $40 \mathrm{~mm}^{2}$ of active cortex. Linear 3D distances between the neuronal sources of the thumb and the little finger were found to lie 1.1 to $2 \mathrm{~cm}$ apart. $23,37,38$

The spatial resolution of MEG depends additionally on the depth of the neuronal source. ${ }^{46}$ Repeated trials in a healthy volunteer demonstrated the reproducibility of MS imaging somatosensory localizations with a test-retest repeatability of $6.7 \mathrm{~mm}$ for one standard deviation. ${ }^{16}$ Integrating the image data set into a neuronavigation system further influences the intraoperative localization accuracy of MS imaging. Typically, the mean localization error of the navigation system ranges between 1.5 and $4 \mathrm{~mm} \cdot{ }^{19,21,54}$ At the time of dural opening an additional error on the order of 5 $\mathrm{mm}$ can be expected due to brain shift. ${ }^{12}$

\section{Comparison of MS Imaging and Intraoperative ECM}

One of the main goals of preoperative functional mapping of the somatosensory cortex is accurate localization of the central sulcus with respect to a space-occupying, intraaxial lesion. Correlations between the displayed functional neuronal sources and anatomical landmarks are helpful to
TABLE 5

Overall patient outcome*

\begin{tabular}{lc}
\hline \hline \multicolumn{1}{c}{ Change in Neurological Status } & No. of Patients (\%) \\
\hline improved & $13(6.5)$ \\
no new deficit/unchanged & $134(66.7)$ \\
new transient deficit & $16(8)$ \\
new deficit but improvement prior to discharge & $23(11.4)$ \\
new permanent mild deficit & $9(4.5)$ \\
new considerable deficit w/ no change prior to discharge & $6(3)$ \\
total & $201(100)^{*}$ \\
\hline
\end{tabular}

* All new deficits were recorded. Data on changes in neurological status were not available in 20 patients, and three patients in this series did not undergo surgery.

check the validity of the MS imaging localization if discrimination of the landmarks is possible on high-resolution MR images. In patients harboring brain tumors encroaching on the rolandic cortex, the anatomy has often been seen to be considerably distorted. A preoperative MR imagingbased definition of the central sulcus, for example, can be hampered in as many as half of the cases. ${ }^{25,29,47}$

Intraoperative ECM has been recognized as the surgical gold standard for preserving neurological function while operating in the vicinity of functional cortical and subcortical pathways. ${ }^{5-7,30,40,61}$ Therefore, noninvasive functional mapping procedures are usually compared with intraoperative ECM to validate their accuracy. There have been several studies in which authors focused on the qualitative comparison of intraoperative ECM to functional MR imaging ${ }^{14,26,32,42,43,49,53,54,56}$ and to MS imaging. ${ }^{19,20,35,45}$ To the best of our knowledge, however, a direct quantitative comparison of ECM sites and MS imaging source localizations in a larger group of patients has not yet been published. McDonald and colleagues ${ }^{35}$ proposed such a quantitative correlation across mapping modalities and presented a couple of instructive cases. In five patients, Mäkelä, et al. ${ }^{33}$ measured nine distances between corresponding MS imaging source localizations and intraoperative ECM sites. In a 3D model, they projected the MS imaging source localization to the surface of the corresponding gyrus. The localization of the ECM site was captured on an intraoperative photograph for postoperative comparison. The mean distance between the MS imaging source localization and the site producing a sensory response during cortical stimulation was $8 \mathrm{~mm}$, and the distance between the MS imaging source localization and the somatosensory evoked potential grid electrode was $10 \mathrm{~mm}$.

Ganslandt, et al. ${ }^{20}$ verified that the use of MS imaging could help correctly to identify the central sulcus within an error margin of approximately $6 \mathrm{~mm}$. They projected one of the MEG dipole source localizations in a two-step procedure onto the central sulcus and then measured the distance to a point derived from a somatosensory evoked potential phase reversal. For comparison, note that Roux, et al.," found in a series of 12 patients a mean distance of $7.4 \mathrm{~mm}$ between the epicenters of the activated area on functional MR imaging and the area found on performing intraoperative ECM. In a set of five patients, Yousry, et al.. ${ }^{64}$ reported that the distance between sites on functional MR imaging and those on intraoperative ECM to be less than $10 \mathrm{~mm}$.

In our study, we found a slightly larger mean distance than has been reported in the aforementioned smaller stud- 


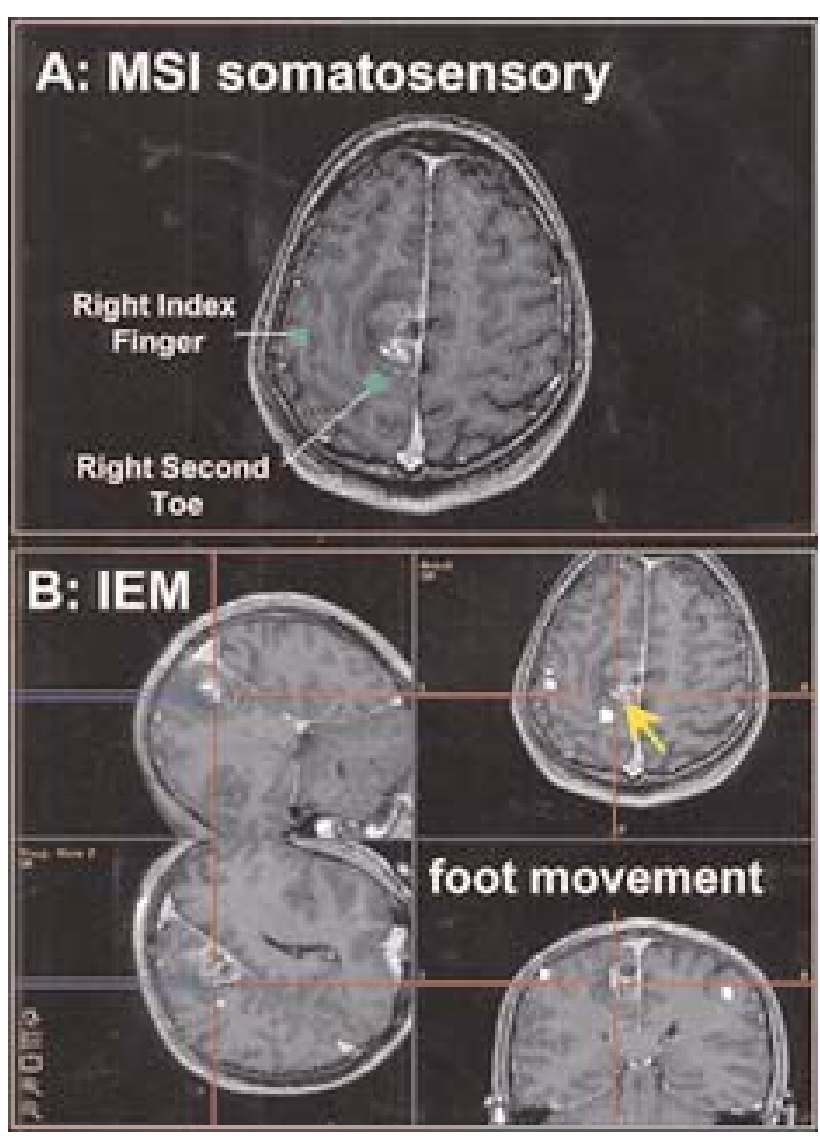

FIG. 2. Case 2. This 38-year-old man presented with a motor deficit and a mild tremor in his right lower extremity. An MR image revealed a contrast-enhancing lesion in the left supplementary motor area. Resection of the glioblastoma multiforme was performed after completing cortical and subcortical electrophysiological mapping while the patient was under general anesthesia. The patient experienced no new postoperative neurological deficit and was discharged from the hospital in a stable condition on the third postoperative day. A: A $T_{1}$-weighted MR image of SED modeled sources (green square and circle) corresponding to tactile stimulation of the right index finger and right second toe. B: Intraoperative neuronavigation system snapshots displaying a subcortical stimulation site that elicited movement of the foot. Note the proximity of the preoperatively acquired MS imaging source localization across the central sulcus to the intraoperative subcortical stimulation site (axial view, upper right panel). These two points were not included in the calculation on the intermodality correlation of cortical sites, but are displayed as an example of the additional value of subcortical mapping.

ies. Four main reasons might account for this discrepancy. First, we retrospectively compared a broad spectrum of motor and somatosensory responses elicited on intraoperative ECM to the MS imaging source localization that matched the anatomical region best. Limitations to a more exhaustive and site-specific comparison included the need for both a standardized, time-efficient, presurgical mapping protocol and the restricted access to cortex during tumor surgery in which the craniotomy was centered to expose primarily the tumor location and the directly adjacent functional cortex. Second, we did not project the MS imaging source localization in a complex 3D model onto the surface, but used the more straightforward mediolateral projection technique, neglecting the anterolateral to the posteromedial course of the central sulcus (Fig. 2) and thus risking the possibility of a small but nonnegligible negative bias in an anteroposterior direction in our comparisons. As such, our estimate of a 12.5-mm displacement between MS imaging and ECM sites may in fact exceed the magnitude of the discrepancy. In fact, it is impossible to identify in all cases the course of the central sulcus, because of the mass effect of a lesion. Third, using a neuronavigation system and screen snapshots for documentation purposes, the much-discussed brain shift problem and the neuronavigation system's registration error were imported into the final equation of distance measurement. Although this factor increased the determined displacement figure, it also offered a more realistic perspective, because making optimal use of preoperative functional mapping data involves incorporating them into the neuronavigation system despite the additional spatial uncertainty incurred. Last, but not least, the intraoperative ECM process itself, during which a bipolar stimulation electrode with 5-mm-spaced tips was used, led to the same responses from stimulation sites an average of $11.3 \mathrm{~mm}$ apart. The major portion of this distance $(8.7 \mathrm{~mm})$ was in the superoinferior direction, as expected from the main course of the rolandic cortex. In the anteroposterior direction a displacement of $4.8 \mathrm{~mm}$ was observed. These findings are in accordance with the widths of the precentral and postcentral gyri, which have been determined from 3D surface reconstructions to be approximately $10 \pm 5$ and $7 \pm 3 \mathrm{~mm}$, respectively. ${ }^{33}$

Given the various contributions to uncertainty in localization comparisons between MS imaging and intraoperative ECM, a distinction must be made between random errors and systematic bias. In fact, the only known systematic displacement in this study is revealed in the two comparisons between somatosensory MS imaging and intraoperative somatosensory mapping and intraoperative motor mapping. In this case, the mean displacement difference was $6.5 \mathrm{~mm}$ in the anteroposterior direction (across the central sulcus). Note that this $6.5 \mathrm{~mm}$ systematic displacement is resolved to be of statistical significance within the data of this group; that is, the MS imaging and ECM methodologies compared within this study do indeed have the ability to resolve a systematic effect on the order of $6.5 \mathrm{~mm}$, despite intrinsic uncertainties associated with the individual methods. It is tempting to speculate that such a systematic bias can indeed be compensated for in subsequent studies in which researchers compare somatosensory MS imaging with intraoperative motor mapping by, for example, simply incorporating an estimated $6.5 \mathrm{~mm}$ anteroposterior localization shift.

In summary, an MS imaging source localization accuracy of $12.5 \mathrm{~mm}$ seems to be a reasonable starting point in the preoperative planning of surgical approaches, resection strategies, and treatment options. At this point, MS imaging should be considered as an aid or adjunct to intraoperative electrophysiological measurements. In addition, in the present group of patients with tumors, the intraoperative subcortical mapping of motor pathways often led to restricting the extent of resection (Fig. 2); thus, we see the next complementary step in preoperative functional imaging as the incorporation of diffusion tensor images, or another method of assessing white matter fibers, into a patient's volume data set to define motor and possibly other subcortical pathways. 


\section{Conclusions}

In conclusion, MS imaging can be considered a valuable and quantitatively accurate planning adjunct in the treatment of patients with intraaxial brain tumors. The use of MS imaging can facilitate clinical decision making by helping to identify patients in whom a safe resection of the intraaxial tumor cannot be accomplished. Furthermore, it can serve as an intraoperative road map for ECM. The combination of preoperative MS imaging and intraoperative ECM allows a radical resection of tumors in highly important functional brain areas with a favorable surgical outcome in the patient.

\section{Acknowledgments}

We are grateful to Susanne Honma, R.T., Mary Mantle, R.EEG.T, Jeff Walker, B.S., and Anthony Leong, B.S., for excellent technical assistance. We also thank Elizabeth Disbrow, Ph.D., for helpful discussions and Prof. Ernst H. Grote for his extraordinary support of international research collaborations.

\section{References}

1. Alberstone CD, Skirboll SL, Benzel EC, et al: Magnetic source imaging and brain surgery: presurgical and intraoperative planning in 26 patients. J Neurosurg 92:79-90, 2000

2. Albert FK, Forsting M, Sartor K, et al: Early postoperative magnetic resonance imaging after resection of malignant glioma: objective evaluation of residual tumor and its influence on regrowth and prognosis. Neurosurgery 34:45-61, 1994

3. Ammirati M, Vick N, Liao YL, et al: Effect of the extent of surgical resection on survival and quality of life in patients with supratentorial glioblastomas and anaplastic astrocytomas. Neurosurgery 21:201-206, 1987

4. Berger MS, Deliganis AV, Dobbins J, et al: The effect of extent of resection on recurrence in patients with low-grade cerebral hemisphere gliomas. Cancer 74:1784-1791, 1994

5. Berger MS, Kincaid J, Ojemann GA, et al: Brain mapping techniques to maximize resection, safety, and seizure control in children with brain tumors. Neurosurgery 25:786-792, 1989

6. Berger MS, Rostomily RC: Low grade gliomas: functional mapping resection strategies, extent of resection, and outcome. J Neurooncol 34:85-101, 1997

7. Black PM, Ronner SF: Cortical mapping for defining the limits of tumor resection. Neurosurgery 20:914-919, 1987

8. Cedzich C, Taniguchi M, Schäfer S, et al: Somatosensory evoked potential phase reversal and direct motor cortex stimulation during surgery in and around the central region. Neurosurgery 38: 962-970, 1996

9. Chapman RM, Ilmoniemi RJ, Barbanera S, et al: Selective localization of alpha brain activity with neuromagnetic measurements. Electroencephalogr Clin Neurophysiol 58:569-572, 1984

10. Danks RA, Aglio LS, Gugino LD, et al: Craniotomy under local anesthesia and monitored conscious sedation for the resection of tumors involving eloquent cortex. J Neurooncol 49:131-139, 2000

11. Daumas-Duport C, Scheithauer B, O'Fallon J, et al: Grading of astrocytomas. A simple and reproducible method. Cancer 62: 2152-2165, 1988

12. Dorward NL, Alberti O, Velani B, et al: Postimaging brain distortion: magnitude, correlates, and impact on neuronavigation. J Neurosurg 88:656-662, 1998

13. Fadul C, Wood J, Thaler H, et al: Morbidity and mortality of craniotomy for excision of supratentorial gliomas. Neurology 38: 1374-1379, 1988
14. Fandino J, Kollias SS, Wieser HG, et al: Intraoperative validation of functional magnetic resonance imaging and cortical reorganization patterns in patients with brain tumors involving the primary motor cortex. J Neurosurg 91:238-250, 1999

15. Freudenstein D, Duffner F, Preiß1 H, et al: Magnetenzephalographie in der Neurochirurgie. Ein Routineprotokoll zur präoperativen Planung und intraoperativen Navigation. Klin Neuroradiol 10:76-84, 2000

16. Gallen CC, Schwartz B, Rieke K, et al: Intrasubject reliability and validity of somatosensory source localization using a large array biomagnetometer. Electroencephalogr Clin Neurophysiol 90: 145-156, 1994

17. Gallen CC, Schwartz BJ, Bucholz RD, et al: Presurgical localization of functional cortex using magnetic source imaging. J Neurosurg 82:988-994, 1995

18. Gallen CC, Sobel DF, Waltz T, et al: Noninvasive presurgical neuromagnetic mapping of somatosensory cortex. Neurosurgery 33: 260-268, 1993

19. Ganslandt O, Fahlbusch R, Nimsky C, et al: Functional neuronavigation with magnetoencephalography: outcome in 50 patients with lesions around the motor cortex. J Neurosurg 91:73-79, 1999

20. Ganslandt O, Steinmeier R, Kober H, et al: Magnetic source imaging combined with image-guided frameless stereotaxy: a new method in surgery around the motor strip. Neurosurgery 41: 621-628, 1997

21. Gumprecht HK, Widenka DC, Lumenta CB: BrainLab VectorVision Neuronavigation System: technology and clinical experiences in 131 cases. Neurosurgery 44:97-105, 1999

22. Hämäläinen M, Hari R, Ilmoniemi RJ, et al: Magnetoencephalography - theory, instrumentation, and applications to noninvasive studies of the working human brain. Rev Mod Phys 65:413-497, 1993

23. Hari R, Karhu J, Hämäläinen M, et al: Functional organization of the human first and second somatosensory cortices: a neuromagnetic study. Eur J Neurosci 5:724-734, 1993

24. Hund M, Rezai AR, Kronberg E, et al: Magnetoencephalographic mapping: basis of a new functional risk profile in the selection of patients with cortical brain lesions. Neurosurgery 40:936-943, 1997

25. Inoue $\mathrm{T}$, Shimizu $\mathrm{H}$, Nakasato $\mathrm{N}$, et al: Accuracy and limitation of functional magnetic resonance imaging for identification of the central sulcus: comparison with magnetoencephalography in patients with brain tumors. Neuroimage 10:738-748, 1999

26. Jack CR Jr, Thompson RM, Butts RK, et al: Sensory motor cortex: correlation of presurgical mapping with functional MR imaging and invasive cortical mapping. Radiology 190:85-92, 1994

27. Kamada K, Takeuchi F, Kuriki S, et al: Functional neurosurgical simulation with brain surface magnetic resonance images and magnetoencephalography. Neurosurgery 33:269-273, 1993

28. Keles GE, Lamborn KR, Berger MS: Low-grade hemispheric gliomas in adults: a critical review of extent of resection as a factor influencing outcome. J Neurosurg 95:735-745, 2001

29. Kober H, Nimsky C, Möller M, et al: Correlation of sensorimotor activation with functional magnetic resonance imaging and magnetoencephalography in presurgical functional imaging: a spatial analysis. Neuroimage 14:1214-1228, 2001

30. Kombos T, Suess O, Ciklatekerlio Ö, et al: Monitoring of intraoperative motor evoked potentials to increase the safety of surgery in and around the motor cortex. J Neurosurg 95:608-614, 2001

31. Kristeva-Feige R, Walter H, Lutkenhoner B, et al: A neuromagnetic study of the functional organization of the sensorimotor cortex. Eur J Neurosci 6:632-639, 1994

32. Lehéricy S, Duffau H, Cornu P, et al: Correspondence between functional magnetic resonance imaging somatotopy and individual brain anatomy of the central region: comparison with intraoperative stimulation in patients with brain tumors. J Neurosurg 92: 589-598, 2000

33. Mäkelä JP, Kirveskari E, Seppä M, et al: Three-dimensional inte- 
gration of brain anatomy and function to facilitate intraoperative navigation around the sensorimotor strip. Hum Brain Mapp 12: 180-192, 2001

34. Matz PG, Cobbs C, Berger MS: Intraoperative cortical mapping as a guide to the surgical resection of gliomas. J Neurooncol 42: 233-245, 1999

35. McDonald JD, Chong BW, Lewine JD, et al: Integration of preoperative and intraoperative functional brain mapping in a frameless stereotactic environment for lesions near eloquent cortex. Technical note. J Neurosurg 90:591-598, 1999

36. Meyer FB, Bates LM, Goerss SJ, et al: Awake craniotomy for aggressive resection of primary gliomas located in eloquent brain. Mayo Clin Proc 76:677-687, 2001

37. Mogilner A, Grossman JA, Ribary U, et al: Somatosensory cortical plasticity in adult humans revealed by magnetoencephalography. Proc Natl Acad Sci USA 90:3593-3597, 1993

38. Nakamura A, Yamada T, Goto A, et al: Somatosensory Homunculus as drawn by MEG. Neuroimage 7:377-386, 1998

39. Nakasato N, Yoshimoto T: Somatosensory, auditory, and visual evoked magnetic fields in patients with brain diseases. J Clin Neurophysiol 17:201-211, 2000

40. Ojemann G, Ojemann J, Lettich E, et al: Cortical language localization in left, dominant hemisphere. An electrical stimulation mapping investigation in 117 patients. J Neurosurg 71:316-326, 1989

41. Penfield W, Boldrey E: Somatic motor and sensory representation in the cerebral cortex of man as studied by electrical stimulation. Brain 60:389-443, 1937

42. Puce A, Constable RT, Luby ML, et al: Functional magnetic resonance imaging of sensory and motor cortex: comparison with electrophysiological localization. J Neurosurg 83:262-270, 1995

43. Pujol J, Conesa G, Deus J, et al: Presurgical identification of the primary sensorimotor cortex by functional magnetic resonance imaging. J Neurosurg 84:7-13, 1996

44. Rezai AR, Hund M, Kronberg E, et al: The interactive use of magnetoencephalography in stereotactic image-guided neurosurgery. Neurosurgery 39:92-102, 1996

45. Roberts TPL, Ferrari P, Perry D, et al: Presurgical mapping with magnetic source imaging: comparisons with intraoperative findings. Brain Tumor Pathol 17:57-64, 2000

46. Roberts TPL, Rowley HA: Magnetic source imaging as a tool for presurgical functional brain mapping. Neurosurg Clin N Am 8: 421-438, 1997

47. Roberts TPL, Zusman E, McDermott M, et al: Correlation of functional magnetic source imaging with intraoperative cortical stimulation in neurosurgical patients. J Image Guid Surg 1:339-347, 1995

48. Roux FE, Boulanouar K, Ranjeva JP, et al: Cortical intraoperative stimulation in brain tumors as a tool to evaluate spatial data from motor functional MRI. Invest Radiol 34:225-229, 1999

49. Roux FE, Ibarrola D, Tremoulet M, et al: Methodological and technical issues for integrating functional magnetic resonance imaging data in a neuronavigational system. Neurosurgery 49: 1145-1157, 2001

50. Sartorius CJ, Berger MS: Rapid termination of intraoperative stim- ulation-evoked seizures with application of cold Ringer's lactate to the cortex. Technical note. J Neurosurg 88:349-351, 1998

51. Sarvas J: Basic mathematical and electromagnetic concepts of the biomagnetic inverse problem. Phys Med Biol 32:11-22, 1987

52. Schiffbauer H, Ferrari P, Rowley HA, et al: Functional activity within brain tumors: a magnetic source imaging study. Neurosurgery 49:1313-1321, 2001

53. Schlosser MJ, Luby M, Spencer DD, et al: Comparative localization of auditory comprehension by using functional magnetic resonance imaging and cortical stimulation. J Neurosurg 91: 626-635, 1999

54. Schulder M, Maldjian JA, Liu WC, et al: Functional image-guided surgery of intracranial tumors located in or near the sensorimotor cortex. J Neurosurg 89:412-418, 1998

55. Simos PG, Papanicolaou AC, Breier JI, et al: Localization of language-specific cortex by using magnetic source imaging and electrical stimulation mapping. J Neurosurg 91:787-796, 1999

56. Stapleton SR, Kiriakopoulos E, Mikulis D, et al: Combined utility of functional MRI, cortical mapping, and frameless stereotaxy in the resection of lesions in eloquent areas of the brain in children. Pediatr Neurosurg 26:68-82, 1997

57. Taylor MD, Bernstein M: Awake craniotomy with brain mapping as the routine surgical approach to treating patients with supratentorial intraaxial tumors: a prospective trial of 200 cases. J Neurosurg 90:35-41, 1999

58. Weinberg H, Cheyne D, Crisp D: Electroencephalographic and magnetoencephalographic studies of motor function. Adv Neurol 54:193-205, 1990

59. Williamson SJ, Kaufman L, Brenner D: Latency of the neuromagnetic response of the human visual cortex. Vision Res 18: 107-110, 1978

60. Wirtz CR, Knauth M, Staubert A, et al: Clinical evaluation and follow-up results for intraoperative magnetic resonance imaging in neurosurgery. Neurosurgery 46:1112-1122, 2000

61. Woolsey CN, Erickson TC, Gilson WE: Localization in somatic sensory and motor areas of human cerebral cortex as determined by direct recording of evoked potentials and electrical stimulation. J Neurosurg 51:476-506, 1979

62. Yang TT, Gallen CC, Schwartz BJ, et al: Noninvasive somatosensory homunculus mapping in humans by using a large-array biomagnetometer. Proc Natl Acad Sci USA 90:3098-3102, 1993

63. Yingling CD, Ojemann S, Dodson B, et al: Identification of motor pathways during tumor surgery facilitated by multichannel electromyographic recording. J Neurosurg 91:922-927, 1999

64. Yousry TA, Schmidt UD, Jassoy AG, et al: Topography of the cortical motor hand area: prospective study with functional MR imaging and direct motor mapping at surgery. Radiology 195: 23-29, 1995

Manuscript received January 4, 2002.

Accepted in final form August 20, 2002.

Address reprint requests to: Timothy P. L. Roberts, Ph.D., Department of Medical Imaging, University of Toronto, 150 College Street, Room 88, Toronto, Ontario M5S 3E2, Canada. email: Tim. Roberts@utoronto.ca. 